Doug Geisler, Eva K. Grebel, and Dante Minniti, eds.

\title{
An HST View of 30 Doradus
}

Jesús Maíz-Apellániz

Nolan R. Walborn

STScI, 3700 San Martin Drive, Baltimore, MD 21218, USA

Rodolfo H. Barbá

Univ. Nacional de la Plata, Paseo del Bosque S/N, La Plata, Argentina

\begin{abstract}
We present a five-field, six-color WFPC2 mosaic of 30 Doradus which constitutes the most detailed ever optical image of a starburst region. With the aid of NICMOS, STIS, and CO data we discuss the most recent star formation history in the region and its relationship with the original and present structure of the parent molecular cloud.
\end{abstract}

\section{Description}

We have combined HST/WFPC2 data from several programs to produce a sixfilter mosaic (F336W, WFPC2 $U$; F555W, WFPC2 $V$; F814W, WFPC2 $I$; F673N, [S II] $\lambda 6717+6731 ; \mathrm{F} 656 \mathrm{~N}, \mathrm{H} \alpha$; and F502N, [O III] $\lambda 5007)$ of the central $70 \mathrm{pc} \times 45 \mathrm{pc}$ of the 30 Doradus nebula at $0 ! 1(0.025 \mathrm{pc})$ resolution. We also analyze more detailed (up to 0.03 or $0.008 \mathrm{pc}$ resolution) images of four regions: knot 3 , R136, knot 1 , and field 15 . The enlargements were produced from the same data sets used to produce the large mosaic as well as from NICMOS data from HST program 7819. Finally, STIS optical spectrograms of the stars in knots 1, 2, and 3 obtained under HST program 8163 were also used. The images can be retrieved from http://www.stsci.edu/ ${ }^{\sim}$ jmaiz.

\section{The overall structure}

Several stellar components are visible in the WFPC2 mosaic. Near the center is R136, which is an extremely compact core, even by super star cluster standards, with a half-light radius of only 0.40 pc. R136 is surrounded by a thick halo which fills a large fraction of the field of view and contains most of the young stars in 30 Doradus. The innermost part of the halo is visible around R136 while the younger, outermost part is still embedded in the surrounding nebulosity. A third population of 4-6 Myr old OB supergiants is spatially mixed with the first two and is not easily identified in the mosaic. Finally, a $\sim 10$ Myr cluster (Hodge 301) is located near the NW corner (Walborn \& Blades 1997).

The nebular structure is more asymmetric. High-excitation gas is seen mostly at a shell located at $10-20$ pc from R136 towards the N and W. Low- 
excitation gas is more uniformly spread over the field but is more concentrated behind (as seen from R136) the high excitation gas. The physical explanation for this morphology is provided by the Johansson et al. (1998) data: at the W and NE sides of our field of view there are two large molecular clouds which are joined by a lower-intensity $\mathrm{CO}$ bridge corresponding to the yellow-green area in our mosaic. The high-excitation gas originates when the radiation from R136 and its surrounding halo strikes the molecular cloud, first photodissociating the gas and then ionizing it. The low-excitation emission originates at the borders of the two molecular clouds not directly visible from R136 and its inner halo, and thus subject to a more diffuse ionizing radiation field. The original molecular cloud must have been much larger, filling most of the field of view, but the birth of R136 and its halo has eroded it in the past $2 \mathrm{Myr}$ to its present size and shape. In another 2-3 Myr the whole cloud will probably be destroyed by the ionizing radiation and winds from the massive stars in the region.

\section{The new generation}

The high excitation shell is ionized not only from the central source but also from within by the second generation of massive stars being produced there. Many 1-2 pc long pillars are visible here, all pointing towards R136. The evolution of these pillars can be seen comparing the knot 3 and 1 regions. In the lower knot 3 region pillar no IR source is yet apparent; if there is a protostar inside it must still be a class 0 object. The two sources in the upper pillar have already passed this stage and they appear as two bright IR sources, weakly visible in the optical. Finally, the sources in knot 1 are full-blown $\mathrm{O}$ and early B stars which are in the process of destroying their cocoon-pillar by carving a cavity around them and removing its top.

Farther away from R136 more young objects are found still embedded in the molecular cloud. Eight pc to the NE of knot 1 is knot 2, an O3-4 star surrounded by several IR sources (Walborn et al. 1999). Another $8 \mathrm{pc}$ to the $\mathrm{N}$ of knot 2 we find an IR cluster with some stars only barely visible in our F814W image and some not even detected there. The cluster must be well embedded in the molecular cloud because no high-excitation gas is detected in the optical image.

Massive young clusters are produced in a variety of morphologies (MaízApellániz 2002). Super star clusters have the same structure as 30 Doradus: a compact core surrounded by a massive halo. Our analysis of 30 Doradus strongly suggests that such clusters are formed inside-out, with the core (and maybe the inner halo) being $\sim 2$ Myr older than the outer halo.

\section{References}

Johansson, L. E. B. et al. 1998, A\&A, 331, 857

Maíz-Apellániz, J. 2002, these proceedings

Walborn, N. R. \& Blades J. C. 1997, ApJS, 112, 457

Walborn, N. R. et al. 1999, AJ, 117, 225 


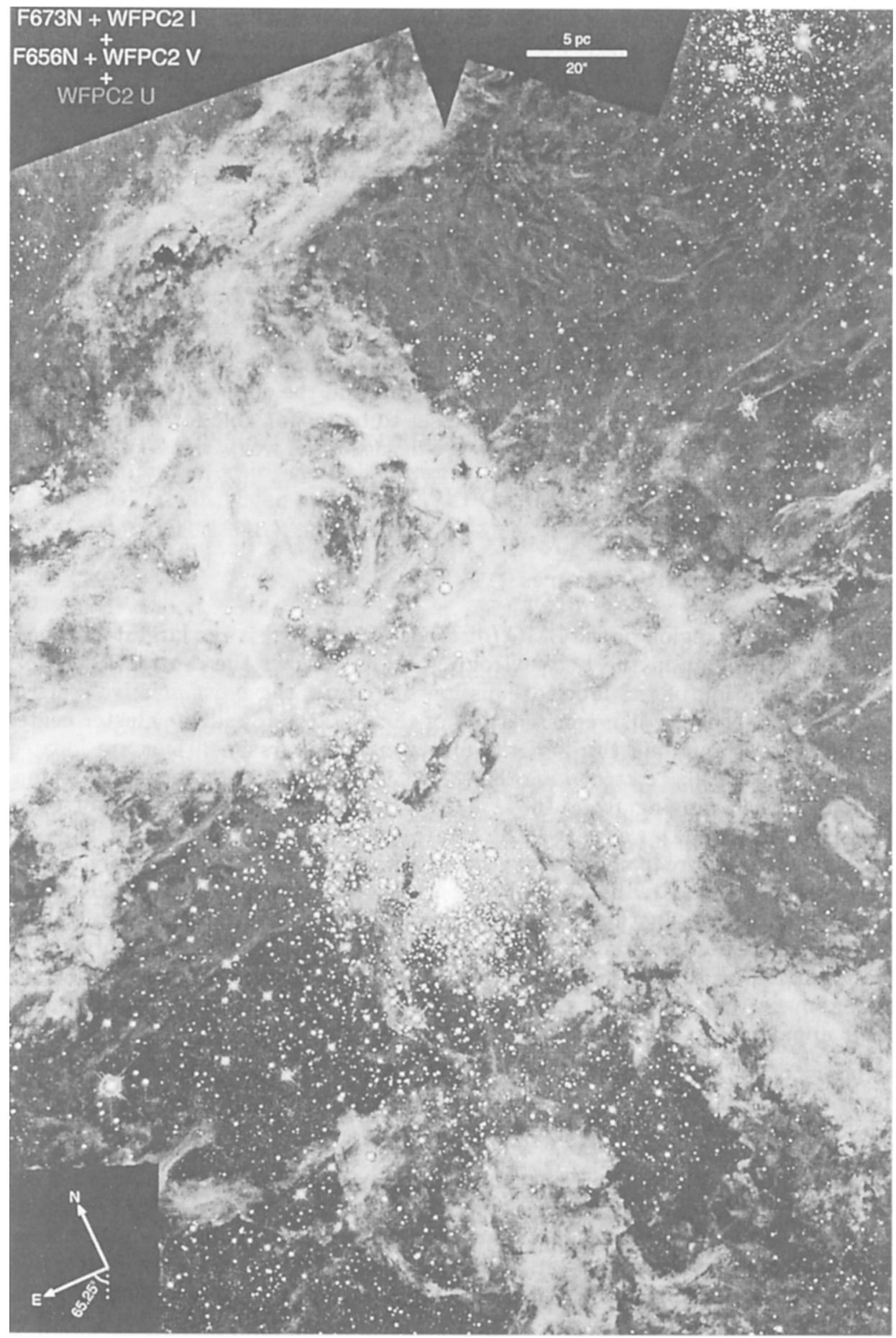

Figure 1. The 30 Doradus WFPC2 mosaic. 